\title{
PRESENTACIÓN
}

\section{EDUCACIÓN MEDIÁTICA EN IBEROAMÉRICA: DESARROLLO Y DESAFÍOS}

El número 32 de Contratexto es publicado casi a la par que el libro Media Education in Latin America (Mateus, Andrada y Quiroz, 2019), pensado como un estado de la cuestión regional y publicado en inglés para promover el diálogo con la comunidad anglosajona. Para nosotros, como editores, es un gran honor contribuir a la reflexión sobre las relaciones que se dan entre la educación y la comunicación con dos publicaciones emparentadas por su concepción y propósitos. En ambos casos, la idea es profundizar en un campo de estudio cuya historia en nuestra región podemos rastrear desde hace unos 50 años (Soares, 2019).

A pesar de su longevidad, la educación mediática, educomunicación o media education mantiene una deuda importante en nuestros países al no haber tomado forma de proyectos y políticas educativas sostenidas en el tiempo. Las más de las veces, el enlace entre educación y tecnologías se ha limitado a la finalidad instrumental expresada en la compra de dispositivos, sin considerar la lógica del acceso a un derecho fundamental. La educación mediática es un sistema que desarrolla capacidades críticas y creativas de interacción entre ciudadanos y medios de comunicación, que incluyen de modo convergente los llamados masivos o tradicionales y los nuevos medios digitales o tecnologías de la información y de la comunicación (TIC). Más allá de sus denominaciones, se trata de un campo interdisciplinar que ha recuperado vigencia en los últimos años en el contexto de una excesiva oferta informativa y el tráfico de noticias falsas que afecta abiertamente la convivencia democrática. En este contexto, el mundo ha vuelto su mirada sobre la educación mediática como una posible salida al problema (Bulger y Davison, 2018).

Pero es justo recordar que esta demanda no es reciente. El famoso informe de Unesco Un solo mundo, voces múltiples: comunicación e información en nuestro tiempo, señalaba en 1980 que las relaciones entre la educación y la comunicación venían multiplicándose como producto de la extensión de los medios, especialmente del audiovisual (International Commission for the Study of Communication Problems, 1980, pp. 56-57). Por otro lado, la Declaración de Grünwald de 1982, hito fundacional 
del movimiento de educación mediática a nivel global, advirtió que los sistemas político y educativo debían comprometerse a reconocer como una obligación la promoción de proyectos que apunten al desarrollo de una actitud más crítica frente a los medios (Unesco, 1982). Más recientemente, la Unesco (2016) ha vuelto a la carga proponiendo a sus estados miembros hacerse cargo de programas y políticas de alfabetización mediática e informacional (AMI) bajo un nuevo enfoque integrador.

La finalidad es cualificar la participación cívica en la vida democrática en un entorno cada vez más mediatizado. En este sentido, la Comisión y el Parlamento Europeo promueven la educación mediática y discuten en el ámbito educativo su escolarización formal y, en el ámbito mediático, su incorporación como tarea de los organismos regulatorios (Frau-Meigs, Velez y Flores-Michel, 2017). Sin embargo, la sensación de algunos expertos, a pesar de los esfuerzos, es de estancamiento y desencanto (Buckingham, 2018).

En América Latina cada país constituye un universo propio, por lo que ha sido difícil promover un desarrollo más orgánico de la educación mediática en el ámbito regional. Esto no significa que las tecnologías hayan estado al margen de las escuelas. Todo lo contrario: Latinoamérica es una de las regiones más dinámicas en términos de compra de tecnología y son notables los esfuerzos por superar las brechas de acceso (Lugo, Kelly y Schurmann, 2015). Sin embargo, estas acciones rara vez son sustentadas bajo el enfoque de la educación mediática al que nos referimos.

Este número de Contratexto recibió propuestas de autores y autoras de países iberoamericanos (Argentina, Ecuador, México, Brasil, España, Honduras, Perú y Venezuela), las cuales fueron revisadas por expertos de distintas partes del mundo. Finalmente, fueron aceptados 13 trabajos del número temático, a los que se suma un texto en la sección Tendencias.

Iniciamos con dos estudios multinacionales. En el primero de ellos, Mittzy Arciniega y Mónica Figueras-Maz realizan una reflexión sobre el uso de los recursos digitales en el aula y su relación con la educación mediática en los sistemas educativos iberoamericanos. Luego, Manuel Antonio Conde, Kevin Paúl Pullaguari-Zaruma y Oscar Andrés Prada-Espinel analizan las competencias mediáticas de doce candidatos a presidentes en España, Ecuador y Colombia.

Un segundo grupo de contribuciones explora los ámbitos nacionales de Argentina y Ecuador. En el caso argentino, Francisco Albarello y Amalia Hafner analizan documentos oficiales sobre la inclusión de la programación y la robótica en la educación formal como una variante de la educación sobre medios y tecnologías. En el caso de Ecuador, Catalina González-Cabrera, Cecilia Ugalde, Lorena Piedra y Jaime Loja comparan las diferencias en las competencias mediáticas de establecimientos de zonas urbanas, rurales, educación flexible y a distancia. 
Esta edición refleja el hecho histórico de que las experiencias locales son las más abundantes en nuestro continente. Mônica Fantin y Bárbara Malcut dan cuenta de la intervención didáctica a partir del teatro con la metodología de los episodios de aprendizaje situado con jóvenes estudiantes de una clase del octavo año de una escuela pública de Florianópolis, Brasil.

Continuamos con dos investigaciones argentinas. Silvana lovanna Caissón analiza un programa de televisión realizado por niños, niñas y jóvenes en canales de televisión comunitarios. Nora Llaver y Bettina Martino construyen las representaciones de profesores de la asignatura de Comunicación Social de escuelas secundarias en la provincia de Mendoza, y analizan y resignifican los contenidos del Diseño Curricular Provincial de la misma asignatura.

Concluimos esta parte dedicada a lo local con dos estudios provenientes de México. Genaro Aguirre Aguilar reflexiona sobre una experiencia en el estado de Veracruz, México, en la que se muestra la vulnerabilidad en el consumo y uso de las TIC que tienen sus ciudadanos. Rodrigo Zárate-Moedano, Angélica Hernández y Károly Méndez Tello describen una experiencia educativa innovadora realizada en el curso de Gramática del Cine Sonoro, en la especialización de Estudios Cinematográficos de la Universidad Veracruzana.

La quinta parte la hemos dedicado a las tecnologías de la información y de la comunicación y la comunicación digital. Sebastián Novomisky, Gladys Manccini Gisela Assinnato y Adriana Coscarelli visibilizan las posiciones de 45 profesores de la Universidad Nacional de La Plata (UNLP), Argentina, ante la innovación digital y las confrontan con propuestas de actualización profesional. Yaimar Del Valle Montoya González y María Helena Bonilla, a través de un enfoque hermenéutico y de inspiración etnográfica, exploran los usos que las familias atribuyen a la computadora portátil Canaimita, incorporada en los espacios educativos públicos de Venezuela.

Finalizamos la sección Dosier con el texto de Cinthia Margarita Sabillón Jiménez y María Helena Bonilla, quienes analizan y discuten los resultados de una investigación de maestría en educación, en la que se estudiaron las prácticas pedagógicas con tecnologías digitales.

Para concluir con el estudio monográfico, incluimos la entrevista de Pablo Andrada a Valerio Fuenzalida, uno de los investigadores más importantes de la educación sobre medios de comunicación en Latinoamérica, quien realiza un recorrido desde sus primeros estudios en televisión en la década de 1970 hasta sus investigaciones y propuestas actuales sobre la televisión infantil.

Asimismo, presentamos las reseñas de los libros Media Education in Latin America (Mateus, Andrada y Quiroz, 2019), por Diana Rivera-Rogel; Ficciones cercanas: televisión, narración y espíritu de los tiempos (Cappello, 2018), por Marta Lopera, y APPS4CAV 
Creación audiovisual con dispositivos móviles (Suárez, Grané y Tarragó, 2019), por Vanessa Perales Linares.

Por último, en la sección Tendencias, José Rivera analiza cómo se adapta el periodismo especializado en salud a la interactividad, hipertextualidad, multimedialidad e inmediatez del ciberperiodismo a través de entrevistas en profundidad a diez periodistas de cuatro cibermedios mexicanos.

Esperamos que este número contribuya a visualizar la relevancia de la educación mediática y permita un mayor diálogo entre los investigadores de nuestra región. Estamos seguros de que este aporte sumará para impulsar el campo de la educomunicación y establecer diálogos complementarios, simétricos y fecundos con otras realidades.

Julio-César Mateus

Editor adjunto (sección Dosier)

Universidad de Lima

Pablo Andrada

Editor adjunto (sección Dosier)

Universidad Central de Chile

\section{REFERENCIAS}

Buckingham, D. (2018). Media literacy policy in Europe: Where are we going? Recuperado de https://davidbuckingham.net/2018/05/18/media-literacy-policy-in-europewhere-are-we-going/

Bulger, M. y Davison, P. (2018). The promises, challenges, and futures of media literacy. Journal of Media Literacy Education, 10(1), 1-21.

Cappello, G. (Ed.) (2018). Ficciones cercanas: Televisión, narración y espíritu de los tiempos. Lima: Fondo Editorial de la Universidad de Lima.

Frau-Meigs, D., Velez, I. y Flores-Michel, J. (2017). Public policies in media and information literacy in Europe. Cross-country comparisons. Nueva York: Routledge. 
International Commission for the Study of Communication Problems [Unesco] (1980). Un solo mundo, voces múltiples: comunicación e información en nuestro tiempo. México D. F.: Fondo de Cultura Económica. Recuperado de https://unesdoc.unesco.org/ ark:/48223/pf0000040066_spa

Lugo, M. T., Kelly, V. E. y Schurmann, S. (2015). Políticas TIC en educación en América Latina: más allá del modelo 1:1. Campus Virtuales, 1(1), 31-42.

Mateus, J. C., Andrada, P. y Quiroz, M. T. (Eds.) (2019). Media Education in Latin America. Londres: Routledge.

Soares, I. (2019). Educommunication landmarks in Latin America: what should be considered in the last 50 years. En J. C. Mateus, P. Andrada y M. T. Quiroz (Eds.), Media Education in Latin America (pp. 185-199). Londres: Routledge.

Suárez, R., Grané, M. y Tarragó, A. (Eds.) (2019). APPS4CAV Creación audiovisual con dispositivos móviles. Colección Transmedia XXI. Barcelona: Learning, Media and social Interaction. Universitat de Barcelona.

Unesco (1982). The Grünwald Declaration on Media Education. Recuperado de http://www. unesco.org/education/pdf/MEDIA_E.PDF

Unesco (2016). Riga recommendations on media and information literacy in a shifting media and information landscape. Recuperado de http://www.unesco.org/new/ fileadmin/MULTIMEDIA/HQ/Cl/Cl/pdf/Events/riga_recommendations_on_ media_and_information_literacy.pdf 